\title{
Localización geográfica de los servicios de salud en un subsistema de asentamientos rurales del Estado de México: un intento de evaluación
}

\section{Carlos Garrocho*}

Este artículo presenta los primeros resultados que constituyen ei avance de un estudio que pretende evaluar la eficiencia de la localización de los servicios de salud del instituto de Salud del Estado de México. Para ello se utiliza un modelo matemático que define los puntos donde debería localizarse la oferta de servicios para minimizar la distancia total y el promedio que deberán recorrer los usuarios del servicio; después se comparan esos resultados con los de la localización real.

\section{Introducción}

\begin{abstract}
El servicio de salud es, quizá, el más básico de todos los servicios. De él puede depender si un recién nacido vive o muere, si sobrevivimos a alguna enfermedad o accidente $y$, en caso de recuperarnos, si recobramos el uso completo de nuestras facultades esenciales o si sufrimos daños permanentes.
\end{abstract}

David M. Smith, Where the grass in greener,

1979.

Definir la asignación espacial de los recursos públicos ha sido uno de los problemas centrales a los que se ha enfrentado la planificación regional. El reconocimiento de que las localidades no funcionan en forma aislada, sino que interactúan siguiendo complejos patrones de interrelación, ha conducido a los planificadores a

* El autor agradece la participación de Juan Gómez Ruiz como auxiliar de investigación, de Lázaro Mejía en la elaboración de los mapas y en la interpretación topográfica de la región de estudio, y de José Antonio Álvarez Lobato y Hugo Soto Barrera en la construcción del programa de cómputo que permitió aplicar el modelo de localización que se utilizó en este trabajo. Todos ellos colaboran en El Colegio Mexiquense. 
buscar la solución del problema desde la perspectiva del análisis de los sistemas de asentamientos (B. Graizbord y C. Garrocho, 1988; C. Garrocho, 1989).

La planeación regional ha reconocido que los sistemas de asentamientos, mediante los cuales se estructura el territorio, tienen que ser un factor de equilibrio económico, social y político. Uno de los problemas clásicos en la planeación regional ha sido definir una jerarquía y distribución espacial de localidades y servicios, capaz de realizar las funciones de distribución y redistribución de un modo coherente con los objetivos sociales. Es decir, encontrar una forma de organización espacial que enfrente el hecho de que los diferentes servicios han de ser suministrados a diferentes escalas geográficas y que la distribución de éstos deberá ser social y territorialmente justa, aunque estos dos conceptos se basen en controvertidos argumentos éticos (D. Harvey, 1977).

Por otro lado, el hecho de que las unidades médicas rurales de bajo nivel jerárquico constituyan el acceso más importante a los niveles más especializados del sistema de salud, y que una adecuada oferta de servicios sea fundamental para el desarrollo rural (y éste, a su vez, del desarrollo nacional) ha generado gran interés en los servicios médicos rurales.

Este documento es el primer avance de un estudio que pretende evaluar, en un subsistema de asentamientos rurales cuya población presenta bajos niveles de bienestar, la localización de las unidades de servicio de salud concentrado del Instituto de Salud del Estado de México, en términos de su accesibilidad física.

Para la evaluación se utiliza un modelo matemático que define los puntos donde la oferta debería localizarse para minimizar la distancia total y promedio que recorrería la demanda para acceder al servicio. Luego, se comparan esos resultados con los que genera el patrón de localización real de las unidades de salud. De esta manera es posible evaluar la actual ubicación de las unidades de servicio en términos de la eficiencia de su localización.

\section{Consideraciones preliminares}

La importancia de las unidades médicas rurales de nivel básico en los sistemas de salud pública ha sido ampliamente reconocida (por ejemplo, D.R. Phillips, 1979, 1981; P. Knox, 1979; A. Joseph y P. Bantock, 1982; F.M. Mburu, 1983). Estas unidades no sólo proporcionan atención médica, sino que juegan un papel de filtro y/o conexión en los niveles más altos del sistema y se han conver- 
tido en el principal oferente de servicios en las zonas rurales. Sin embargo, la oferta de unidades rurales de primer contacto no siempre es suficiente o adecuada para cumplir con el papel que se le ha asignado.

De lo anterior se deduce que disfrutar de un fácil y rápido acceso a las unidades médicas de atención primaria es un aspecto que incide de manera importante en la efectividad del sistema de salud. Por otra parte, el hecho de que la utilización de estas unidades decrezca significativamente conforme se incrementa la fricción de la distancia, justifica el valor que se ha dado a su relación de accesibilidad respecto a la demanda (J. Eyles, y $\mathrm{K}$. Woods, 1983).

Las desigualdades sociales y territoriales en la provisión de servicios médicos han sido consideradas como uno de los principales problemas a resolver en la planificación de los servicios de salud. Una de las desigualdades más claras que existen es la que se presenta entre el campo y la ciudad, lo que refleja la orientación urbana que han tenido la planificación y la educación médica, la atracción que las ciudades ejercen sobre el personal y la infraestructura relacionada con la salud, y el mayor costo que implica la dotación de servicios en las áreas rurales de baja densidad de población.

Sería ingenuo pensar que el sistema de salud por sí solo puede incrementar efectivamente los niveles de salud de la población y reducir sus desigualdades. En todo caso, es una condición necesaria, pero de ninguna manera suficiente para lograrlo.

La salud y los desiguales niveles de salud de la población se generan dentro de toda una estructura social específica y la reflejan (V. Navarro, 1976; L. Doyal, 1979). Por lo tanto, la salud y la enfermedad están relacionadas con una gran diversidad de variables: ingreso, empleo, vivienda, educación, sanidad, aspectos culturales y medio ambiente (P. Townsand y N. Davidson, 1982). Entre éstas, los servicios médicos sólo constituyen otro factor, $\mathrm{y}$ no precisamente el más importante, en la salud de la población.

Por supuesto, la accesibilidad es sólo uno más de los diferentes factores que influyen en la efectividad de los sistemas de salud. Otros elementos importantes son, por ejemplo, la calidad del servicio o la disponibilidad de los recursos materiales y humanos adecuados.

De cualquier manera, la accesibilidad física de los servicios de salud en las zonas rurales ha ocupado un lugar relevante en la planificación regional. Dos argumentos justifican esto: a) la importancia que tienen los servicios de salud y el papel fundamental que juegan en el crecimiento económico y en el desarrollo regio- 
nal y nacional, y, b) la baja densidad de la población rural, que provoca que las distancias entre los puntos de oferta y de demanda causen que el acceso de los consumidores a los servicios médicos sea difícil y costoso, lo que afecta, por un lado, la eficiencia del sistema y, por el otro, el bienestar de la población.

Desde la perspectiva más amplia de la justicia social, las diferencias de accesibilidad entre la población urbana y rural coloca a ésta en una posición de desventaja que es incompatible con los aspectos esenciales de los bienes y servicios públicos o cuasipúblicos (P.A. Samuelson, 1954 y 1955; R.M. Veatch, 1981; G. de Jung y F. Rutten, 1983). No obstante, esta situación de desigualdad en la oferta de un bien, que en México se pretende sea eminentemente público y equitativo, no ha ocupado especialmente la atención de los planificadores regionales de nuestro país (López Acuña, D., 1982; Ward, P., 1986).

La tendencia a diseñar unidades de servicio especializadas, sofisticadas y de gran tamaño, aunada a los avances tecnológicos en medicina (lo que no siempre incrementa su eficiencia), ha generado la necesidad de concentrar espacialmente los servicios de salud: Esta orientación centralizadora contribuye a agravar los problemas de accesibilidad de la población rural a determinados servicios médicos.

Si bien la concentración espacial de los servicios públicos permite generar economías de escala en dotación y mantenimiento, estas supuestas ventajas deberían ser sistemáticamente contrastadas con los costos que impone a la población en términos de reducción de accesibilidad. Éstos, finalmente, incidirán en su ingreso real $\mathrm{y}$, por tanto, en su bienestar y en la justicia organizativa de la sociedad. En este trabajo se trata, precisamente, de evaluar la localización actual de las unidades de servicios de salud en una zona rural, comparándola con la distribución espacial óptima. Esto permitirá delinear algunos criterios para la ubicación de las nuevas unidades que se incorporen al sistema, en función de los objetivos sociales implícitos en toda oferta de servicios de salud pública.

Necesidad y demanda de servicios de salud

La idea de que la sociedad debería estar organizada para satisfacer las necesidades de todos sus integrantes ha sido un noble sentimiento a lo largo de la historia (S. Pinch, 1985). Marx sugiere que, después de la Revolución, las necesidades serán el criterio que gobierne la producción, distribución y el consumo. Por otra 
parte, la teoría neoclásica argumenta que los consumidores satisfacen sus necesidades adquiriendo los bienes y servicios de acuerdo con sus preferencias. Sin embargo, definir y medir las necesidades humanas ha sido complejo y polémico.

Teóricamente sería posible determinar las necesidades mínimas de sobrevivencia de un individuo, pero el concepto de necesidad es multidimensional. Está relacionado intrínsecamente con las características y condiciones de cada sociedad y responde a normas aceptadas en tiempo y lugares determinados.

No sorprende, entonces, que definir la necesidad y/o demanda de servicios de salud, entendido como un servicio público, ha sido siempre controvertido (R.M. Veatch, 1981). El concepto de necesidad social es inherente a la idea de servicio público y la historia de los servicios públicos es la historia del reconocimiento de las necesidades sociales (J. Bradshaw, 1972).

En un sentido estrictamente económico, la demanda o necesidad de servicios médicos puede ser medida a partir del número de visitas por unidad de tiempo que una población dada realiza al punto de oferta. Pero necesidad significa algo más y está relacionada, cuando menos, con la cantidad y calidad del servicio de salud que las autoridades médicas o que la sociedad crea que debería de tener la población (K. Boulding, 1966; R. Fein, 1967).

Una perspectiva más amplia acerca del concepto de necesidad es la presentada por Bradshaw (J. Bradshaw, 1972), quien distingue cuatro categorías de necesidad: la necesidad normativa, que es definida por los "expertos" o profesionales a partir de la comparación entre lo que ellos creen que es la dotación deseable de un servicio y la oferta existente; la necesidad sentida, que es sinónimo de deseo y es medida por los requerimientos de la población respecto a un servicio específico; la necesidad expresada, que es la necesidad sentida transformada en acción y que se mide por el número de personas que usan o demandan activamente un servicio; y la necesidad comparativa, que se mediría por las diferencias de provisión de un servicio entre grupos de población de características semejantes. El propio Bradshaw afirma, sin embargo, que el concepto de necesidad social no puede ser definido con claridad. Por esta razón, los planificadores de sistemas de salud se han visto obligados a desarrollar sus propias definiciones de necesidad y/o demanda (R. Stimnson, 1981). En todo caso, cualquiera que sea la definición que se utilice, no hay que olvidar que la propensión a usar los servicios de salud variará de acuerdo con las características de la población, así como al acuerdo social del nivel mínimo aceptable de satisfactores que deben existir en un momento dado. 


\section{Principios de distribución de los recursos}

Lograr el más alto nivel de salud para la población es una aspiración que implica un uso y asignación cuidadosos de los recursos humanos (en términos de personal), de capital y de tecnología. El problema tiene dos aspectos principales: por un lado, se tienen que definir los medios y métodos más adecuados para promover la salud. Así, reducir la pobreza, la desnutrición y la insalubridad quizá sea más efectivo que proveer más unidades médicas o que incrementar los niveles tecnológicos de los servicios disponibles (D. López Acuña, 1982). También el otro, habría que reconocer la existencia de desigualdades sociales y de salud, lo que conduce al problema de definir el patrón más adecuado para la distribución de los recursos.

Algunas filosofías políticas proponen principios estructurales que definen en lo general las principales condiciones y objetivos de distribución (por ejemplo: igualdad, equidad, eficiencia). Sin embargo, no dicen nada específico sobre la distribución de bienes y servicios concretos (por ejemplo, el de salud), por lo que ésta tendrá que ser derivada tanto de los principios generales de cada filosofía política como de los objetivos particulares de cada sociedad.

Básicamente se pueden distinguir cuatro modelos que intentan responder al problema de la asignación o distribución de los recursos: el utilitario, el igualitario, el de igualdad de acceso y el de mercado (R.M. Veatch, op. cit.) El modelo utilitario busca optimizar los beneficios netos de la inversión. Su principal ventaja es que maximiza la eficiencia en el uso de los recursos. Sin embargo, la eficiencia no siempre es el valor más importante en las políticas de salud. El modelo igualitario busca homogeneizar tanto como sea posible los niveles de salud de la población. Se fundamenta en el principio de igualdad de resultados, lo que significa que el criterio de distribución es selectivo y que privilegiará a los grupos con menores niveles de salud. Evidentemente, una política de este tipo no puede ser tan eficiente como una política basada en el modelo utilitario. Un tercer modelo descansa en el principio de igualdad de acceso. Este enfoque evita juzgar la efectividad del servicio y las necesidades individuales, lo que le da un sentido más práctico, y busca que toda la población tenga iguales niveles de accesibilidad al servicio, lo que implica que la distribución de la oferta debe tender a igualarse tanto social como territorialmente. De acuerdo con esto, los planificadores centrarán su atención sobre las barreras económicas, sociales y físicas que limitan el acceso de la población a los servicios. La contraparte de este último modelo es el enfoque que se basa en los principios de mercado. Según éste, la 
distribución de los servicios debería ser consecuencia del libre juego de la oferta y la demanda. Los resultados podrán ser desafortunados para ciertos grupos, pero no injustos, dado que "las reglas son iguales para toda la sociedad". ${ }^{1}$ Los que sostienen esta idea no sólo se proclaman los máximos defensores de la libertad individual sino los campeones de la eficiencia (G. de Jung, y F. Rutten, 1983).

\section{El concepto de accesibilidad}

Una de las variables más importantes para definir la localización de los servicios de salud parece ser, pues, su accesibilidad. Ésta depende, fundamentalmente, de la localización del servicio, de la distribución espacial de los consumidores y de la movilidad de ambos (M. Mosley, 1979). En efecto, si se supone constante la calidad interna de un servicio (lo que no siempre es correcto), las diferencias de accesibilidad representan costos extraordinarios para algunos consumidores. La provisión de cualquier servicio (sin importar su tipo) considera, aunque sea implícitamente, su accesibilidad y trata de eliminar toda barrera que dificulte la relación entre su oferta (producción) y su demanda (consumo). Dado que la cantidad y calidad que se puede disfrutar de un servicio público están relacionadas, entre otras cosas, con su accesibilidad, ésta incidirá directamente en la redistribución social del ingreso y, por tanto, en el bienestar y la calidad de vida de la población.

De acuerdo con lo anterior, uno de los principales objetivos de la planificación y la administración pública sería maximizar la accesibilidad de la población a todos los bienes y servicios públicos y privádos. Esto se lograría situándolos tan próximos a la demanda como fuera posible. El problema, entonces, se reduciría a abatir los costos de traslado (medidos en tiempo, dinero, confort, energía, etc.), tomando en cuenta la red de comunicaciones y el modo de transporte disponible.

Desde esta perspectiva, la localización óptima de un servicio sería aquella que minimice los costos de transporte de los consumidores. Sin embargo, ésta es una reducción eficientista y, como ya se mencionó, la eficiencia no es necesariamente el valor más importante en la planificación de los servicios de salud. Desde un

\footnotetext{
${ }^{1}$ Evidentemente esto no es exacto. Los partidarios del enfoque de mercado pretenden ignorar el principio de igualdad de condición que significa que "todos los competidores deben iniciar la carrera desde el mismo punto, sin ventajas de ningún tipo para nadie". (B.S. Turner, 1986), cosa que no sucede en la realidad.
} 
punto de vista equitativo, la solución óptima sería la que minimice las diferencias entre los costos de transporte de los consumidores. De la elección de una u otra solución, o de una combinación de ambas, depende la distribución de los recursos y, por tanto, el mejor cumplimiento de los objetivos sociales.

Pero el concepto de accesibilidad es complejo de estimar debido, entre otras cosas, a la variedad de formas en que se le define. Hagerstrand (1979) afirma que la accesibilidad tanto a los servicios como a los puntos de trabajo, sólo puede ser entendida en función del tiempo disponible del consumidor, del costo/tiempo de recorrido al punto de oferta y del horario del servicio. El problema para medir la accesibilidad de un servicio se relaciona, entonces, con el de medir, cuando menos, las tres variables mencionadas. Aún más, si la accesibilidad está determinada en algún grado por los costos de transporte, ¿cómo evaluar aspectos tales como el nivel de confort del sistema de transporte, el modo y tipo de transporte utilizado, los congestionamientos de tráfico, la calidad de las vías de comunicación, el número de transbordos. . .?

Son dos las maneras básicas como se ha intentado medir la accesibilidad de las unidades primarias de salud; la primera considera medidas de accesibilidad revelada obtenidas mediante encuestas e información documental; la segunda estima la accesibilidad potencial con base en la localización espacial de la población (demanda potencial) y de las unidades médicas (oferta). Las medidas de accesibilidad basadas en la utilización del servicio están limitadas por la complejidad de la conducta de los consumidores, que a su vez resulta influida por otros factores no bien entendidos, tales como la territorialidad, la importancia del tiempo y el espacio y la percepción y el comportamiento espacial. El segundo criterio de medición es más específico, requiere de menos información y ha resultado más práctico para los planificadores (A. Joseph y D. Phillips, 1984).

Evidentemente, y en cualquier situación, algunos consumidores siempre estarán mejor localizados espacial y financieramente respecto al servicio y enfrentarán menores costos de acceso. Los residentes de las áreas rurales no están en esta situación. Ellos tienen particulares problemas de accesibilidad a los servicios públicos y tienen que pagar más por ello. Generalmente, los puntos de oferta en las áreas rurales están localizados a distancias mucho mayores que en las áreas urbanas. Además, la población rural dispone de menores facilidades de transporte y la red de comunicaciones es insuficiente (I. Askew, 1983).

Estudios en Estados Unidos e Inglaterra han mostrado que la accesibilidad a los servicios en las áreas urbanas es casi diez veces 
menor para quienes utilizan servicios de transporte público que para quienes poseen un automóvil propio y que esta situación se agrava en las zonas rurales (M. Hayes, 1986). Si, además, la población de estas áreas tiene los menores niveles de ingreso y de bienestar, su situación es claramente desventajosa. Una distribución territorial más equitativa de los servicios públicos no incidirá en las razones últimas de esta desigualdad, pero quizá contribuya a reducirla en alguna medida. ${ }^{2}$

Un modelo de localización de servicios de salud en áreas rurales

El problema de definir la ubicación territorial de las unidades médicas difiere de los problemas clásicos de localización, debido a que cada una de ellas constituye un elemento dentro de un sistema funcionalmente integrado. Por esa razón, la localización de este tipo de unidades sólo puede ser apropiadamente concebida desde una perspectiva sistémica (N.B. Teitz, 1968). No es necesario considerar las estrategias de localización de otros oferentes, puesto que en la mayoría de los casos, la oferta alternativa es muy reducida o no existe, y la demanda de servicios de salud no está necesariamente ligada a la de otros bienes y servicios. Esto, aunado a la falta de competencia, apoya la idea de que el punto de localización del servicio se defina principalmente en función de su accesibilidad.

En este trabajo se considera la accesibilidad en términos de distancia (acceslbilidad relativa) y no en términos de oportunidades (sociales, económicas, etc.) de acceso (accesibilidad integral) (D. Ingram, 1971).

El modelo de localización seleccionado para este estudio determina los puntos más accesibles en una región, de acuerdo con el concepto de accesibilidad relativa y a la localización espacial de los puntos de demanda potencial. Por lo tanto, se suponen homogéneos los niveles de ingreso de la población, los modos y sistemas de transporte, la disponibilidad de tiempo del consumidor, el

\footnotetext{
2 Es importante reconocer que las desigualdades no tienen una especial relación con el capitalismo. Si éste reemplazó las desigualdades legales del feudalismo por desigualdades económicas, se puede argumentar que los Estados socialistas (al menos como existen en el bloque soviético) han reemplazado éstas por desigualdades basadas en el poder político asociado al Partido. Por otro lado, sugerir que el status quo solamente puede cambiarse mediante una acción revolucionaria situada en un futuro distante e incierto, es una crítica al capitalismo que, en el fondo, resulta profundamente conservadora (B.S. Turner, 1986).
} 
costo de su tiempo y su necesidad del servicio. Por el lado de la oferta, se suponen homogéneos su calidad y horario de trabajo.

Dado que el objetivo es identificar las localidades más accesibles en la región con el fin de que puedan ser puntos de localización de los servicios, se consideró a la población total de cada localidad o punto i como la demanda potencial Wi por servicios. Al mismo tiempo, ésta es una medida del número de viajes que se pueden generar del punto de demanda i al punto de localización del servicio $j$ en cualquier periodo.

El problema general, entonces, es localizar m servicios que satisfagan la demanda de $\mathrm{n}$ puntos i de demanda (localidades). Estos puntos estarán interconectados por una red de comunicaciones y la distancia por carretera (ponderada por el tipo de camino) será la medida de la longitud de los viajes entre los puntos i y j.

Para reducir al mínimo la longitud agregada de los viajes, cada punto de demanda i deberá ser servido por el punto de oferta j más cercano. La localización de los puntos i respecto a los puntos $j$ será indicada por la variable $a_{i j}$. Esta variable será igual a 1 cuando el servicio j sea el más cercano a la localidad, y será igual a 0 cúando no se cumpla esta condición. Recapitulando, $\mathrm{d}_{i j}$ será la distancia de i a $j ; w_{i} \mathrm{~d}_{i j}$ los viajes potenciales de i a j; y $a_{i j} w_{i} \mathrm{~d}_{i j}$ indicará si el punto de oferta j es el más cercano al punto de demanda i, por lo que el objetivo del modelo será abatir el costo total de recorridos de i a $j$.

La expresión formal del modelo sería la siguiente:

$$
\text { Minimizar: } \sum_{i=1}^{n} \sum_{j=1}^{m} \mathrm{a}_{i j} w_{i} \mathrm{~d}_{i j} \sum_{j=1}^{m}
$$

La red de vías de comunicación -en este caso sólo se consideran carreteras y caminos- será descrita en términos de nodos, interconexiones y distancias. Cada interconexión unirá sólo a dos nodos (localidades) y así podrá ser identificada por los nodos que conecta. Con el fin de considerar en el modelo los diferentes tipos de vías, la longitud de cada conexión será ponderada por un factor según el tipo de carretera o camino y expresada en kilómetros.

Los insumos mencionados especifican el problema y el modelo se operativiza de la siguiente manera:

1. Se construye una matriz de distancias minimas entre todas las localidades consideradas de acuerdo a la red de carreteras y caminos.

2. Estas mínimas distancias se ponderan según el tipo de conexión (de acuerdo con los promedios de velocidad que especifica la Secretaría de Comunicaciones y Transportes para cada tipo de 
camino), se multiplica por la demanda potencial en el punto i $\left(W_{i}\right)$ y se suman.

3. El nodo que presente el valor más bajo de viajes-distancia de i a $j\left(W_{i} d_{i j}\right)$ será el punto de localización más accesible.

Sin embargo, la identificación simultánea de varios puntos de oferta de servicios j no es tan sencilla. Los viajes se generarán de cada punto de demanda $i$ al punto de oferta $j$ más cercano. La evaluación de todas las posibles combinaciones de localización de servicios sería extremadamente laboriosa, aun con el uso de computadoras, ya que implica un algoritmo heurístico diseñado para encontrar la mejor solución al problema. El algoritmo funciona de la siguiente manera:

a) Se selecciona aleatoriamente un grupo de nodos como localización inicial de los servicios.

b) Se hace una estimación del total de viajes de los puntos de demanda i al punto de oferta del servicio j más cercano (según el grupo escogido aleatoriamente).

c) Se sustituye secuencialmente cada uno de los nodos seleccionados por cada uno de los no seleccionados.

d) Si la estimación de las distancias totales para un nuevo nodo es inferior a alguna de las estimaciones realizadas para los nodos de la muestra inicial, se incorporará a la muestra en sustitución de aquel cuya estimación de viajes resultó mayor.

e) Los pasos c y d se repiten para cada uno de los nodos no seleccionados en el grupo inicial hasta que ya no se detecten reducciones significativas en el total de viajes generados por todo el conjunto de localidades. Ésta es la solución final. ${ }^{3}$

\section{La región de estudio}

La región de estudio se localiza al suroeste del Estado de México y se definió con base en criterios de homogeneidad de sus niveles de bienestar y desarrollo (F. Rodríguez, 1988; S. Camposortega y M.A. Mejía, 1987; INEGI-SPP, 1987) y de la oferta de servicios médicos a nivel municipal (SEI, 1986; ISEM, 1988). Está conformada por los siguientes municipios: Almoloya de Alquisiras, Amatepec, Coatepec de Harinas, Otzoloapan, San Simón de Guerrero, Sultepec, Tejupilco, Temascaltepec, Texcaltitlán, Tlatlaya, Zacazona-

\footnotetext{
${ }^{3}$ Una amplia explicación del algoritmo utilizado puede verse en: Oppenheim, N., Applied models in urban and regional annalysis, N. Englewood Cliffs, Prentice Hall, Inc., 1980; G. Rushton, Optimal Location Facilities, N.H. Wentworth, Compress, Inc., 1979.
} 
pan y Zacualpan. Esta región es la más pobre del Estado de México, su economía es predominantemente agrícola de temporal y sus niveles de densidad de población son bajos en relación con el resto de la entidad (cuadro 1; mapa 1).

CUADRO 1

Población y densidad municipal en la región de estudio, 1980

\begin{tabular}{lrc}
\hline Municipio & Población & $\begin{array}{c}\text { Densidad de } \\
\text { población por } \mathbf{k m}^{2}\end{array}$ \\
\hline Almoloya de Alquisiras & 10268 & 67.44 \\
Amatepec & 23101 & 42.73 \\
Coatepec de Harinas & 22406 & 126.82 \\
Otzoloapan & 3419 & 45.59 \\
San Simón de Guerrero & 3678 & 55.57 \\
Sultepec & 19631 & 38.75 \\
Tejupilco & $575 n \%$ & 61.94 \\
Temascaltepec & 153 & 30.59 \\
Texcaltitlán & 560 & 52.04 \\
Tlatlaya & 31752 & 45.87 \\
Zacazonapan & 2098 & 22.72 \\
Zacualpan & 12797 & 64.44
\end{tabular}

Fuente: Estado de México: cuaderno de información para la planeación, INEGI-SPP, 1987.

Funcionalmente, la localidad de Tejupilco (9 228 habs. en 1980) es el único centro oferente de bienes y servicios de importancia regional y constituye la localidad más grande del subsistema de asentamientos de la región. Sin embargo, resulta poco accesible para el resto de la población debido a la baja densidad de vías de comunicación y a la reducida oferta de transporte colectivo. El subsistema de asentamientos está integrado por una localidad de 9228 habs.; tres de 2000 a 4999 ; 28 de 1000 a 1999 y 760 localidades menores de 999 habs. Se trata de un conjunto de asentamientos rurales de alta primacía (en cuanto que la localidad más grande en términos demográficos supera con mucho el tamaño de las que le siguen en la jerarquía de asentamientos), poco complejo en su funcionamiento (en cuanto que un solo asentamiento articula y organiza el funcionamiento de las actividades en la región) y de baja integración funcional (en tanto que las relaciones entre los asentamientos son bajas en intensidad y frecuencia) (C. Garrocho, 1988.).

Topográficamente, la región es accidentada y se observan variaciones de altura que van desde los 1000 hasta 3000 metros so- 
MAPA 1

Región de estudio y red principal de vías de comunicación

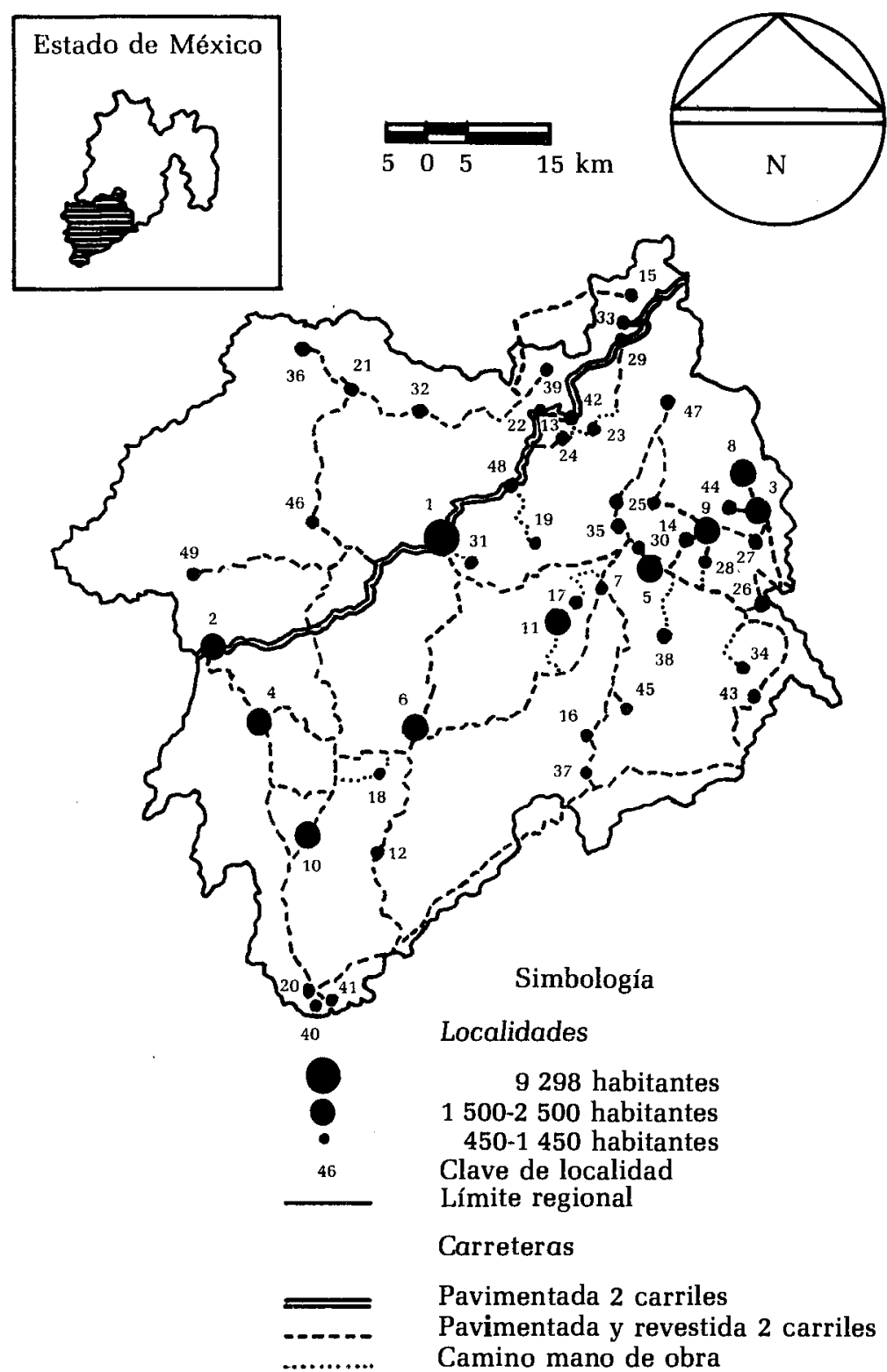


bre el nivel del mar, por lo que la red de caminos que conecta los asentamientos de la región es sinuosa y de baja velocidad.

Resumiendo, diremos que el contexto del presente estudio es el de una región rural, de baja densidad de población, con baja oferta de servicios de salud pública y marginada del desarrollo estatal.

\section{Aplicación del modelo}

En la región de estudio los servicios convencionales de salud son ofrecidos casi en su totalidad por el sistema de salud pública. La participación del sector privado es marginal, aunque las prácticas tradicionales de curanderos y otros agentes relacionados con la oferta de servicios de salud aún son importantes entre la población rural.

El sistema de salud pública en la región de estudio está organizado jerárquicamente y las unidades que lo integran pueden clasificarse en tres niveles: en el nivel más bajo (rango uno) están los centros de servicios de salud rural que no cuentan con servicio de hospitalización y prestan sólo la atención primaria más básica. Son más de 50 y su patrón de localización es disperso. Su efectividad está limitada, entre otras cosas, por la carencia de recursos físicos y humanos y por la irregularidad de su horario de servicio. El siguiente nivel (rango dos) está conformado por seis centros de servicio de salud rural concentrado, que se localizan en las siguientes localidades (mapa 2): Palmar Chico (1 916 habs.), Bejucos (2 468 habs.), Amatepec (1 729 habs.), Luvianos (669 habs.), Temascaltepec (1 385 habs.) y Zacualpan (859 habs.). Estas unidades cuentan con una oferta mayor de servicios incluyendo hospitalización, y articulan funcionalmente el subsistema de salud pública regional, puesto que su disponibilidad de recursos físicos y humanos les dan una mayor formalidad que la que presentan las unidades del nivel básico. Por otro lado, estas unidades juegan un papel preponderante en el sistema como filtros o conexiones entre sus niveles más bajos y los niveles más especializados, lo que incide en la accesibilidad y, por lo tanto, en la eficiencia del sistema de salud. Finalmente, el nivel más alto de la ịerarquía está integrado por un solo elemento: el Hospital General ubicado en Tejupilco (9 228 habs.; mapa 2).

Tomando en cuenta sólo la población de las 50 localidades más grandes en la región (cuadro 2), la red de carreteras y caminos existente y las distancias entre las diferentes localidades, se procedió a calcular, aplicando el modelo expuesto anteriormente, 
MAPA 2

Región de estudio: Rolocalización real y óptima de las unidades de servicio de salud

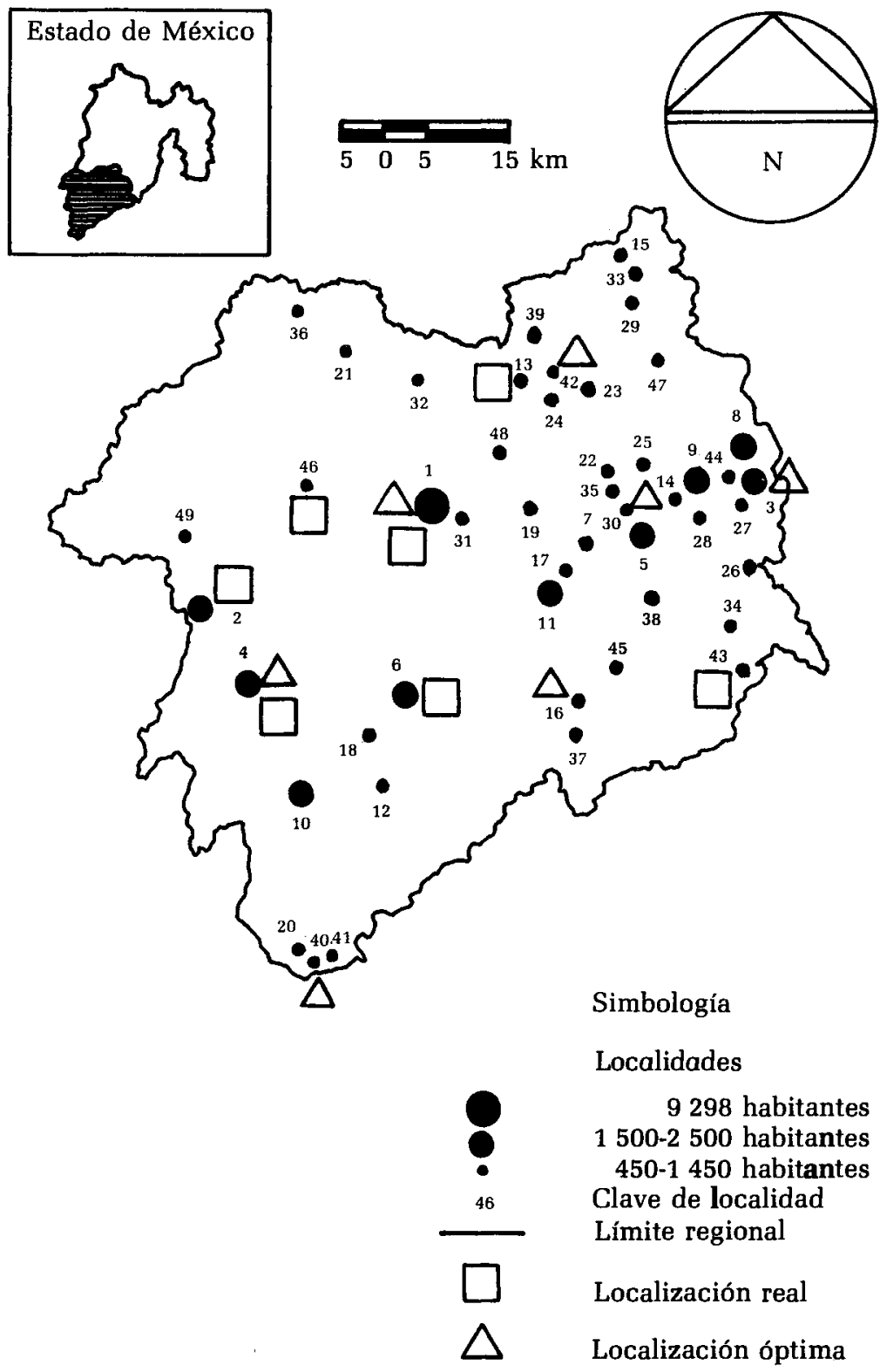


las distancias agregadas y promedio que necesitaría recorrer la demanda potencial para acceder a las unidades de servicio del segundo y tercer nivel, que son las que estructuran el sistema de salud pública en la región (cuadro 3).

Posteriormente, se identificaron los puntos en el territorio que minimizan los recorridos agregado y promedio por habitante de la demanda potencial a las unidades de servicio (el patrón de localización óptima de los elementos del sistema) y, finalmente, se procedió a comparar los recorridos que se generarían en ambos patrones de localización (el "real" y el "óptimo") (cuadro 4; mapa 2).

De acuerdo con los supuestos del modeı, eı punto más accesible en la región es Tejupilco y, por lo tanto, ésa es la localidad que garantiza la máxima eficiencia locacional del único elemento del sistema de salud de rango tres (el Hospital General), lo que coincide con la localización real del hospital.

En cuanto a las seis unidades de servicios de salud rural concentrado, el modelo localiza una de ellas en Palmar Chico (1 916 habs.), lo que coincide con su situación real y otra en Carboneras (864 habs.), localidad ubicada a menos de $5 \mathrm{~km}$ de Temascaltepec, que es donde se localiza realmente una de estas unidades. Las cuatro unidades restantes son ubicadas por el modelo en sitios muy diferentes a los del patrón real: tres de ellas en la parte oeste de la región de estudio, que es la que tiene mayor densidad de asentamientos (en San Andrés Tepetitlán 1001 habs., Coatepec de Harinas 2362 habs. y San Pedro Hueyahualco, 1240 habs., respectivamente), y la última en el extremo sur, casi en el límite estatal (El Naranjo, 892 habs.), donde se localizan otros dos asentamientos importantes, próximos entre sí pero aislados del resto de la región (San Antonio del Rosario 1179 habs., y Nuevo Copaltepec 868 habs.; mapa 2).

Las diferencias mencionadas entre los patrones de localización real y "óptimo" (de acuerdo con los supuestos del modelo), tienen importantes implicaciones en términos de accesibilidad. El recorrido agregado (la población total multiplicada por la distancia que debe cubrir para acceder a la unidad de servicio más próxima) que se deriva del patrón de localización óptimo representa $52.2 \%$ del que se genera en el patrón de localización real y en términos absolutos se está hablando de un ahorro de $1178519 \mathrm{~km}$ de recorrido agregado total para toda la población. El patrón real de localización requiere de un recorrido promedio por habitan- 
CUADRO 2

Localidades de la región de estudio consideradas en el análisis y población, 1980

\begin{tabular}{|c|c|c|}
\hline Clave & Municipio/Localidad & Población \\
\hline 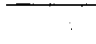 & Almoloya de Alquisiras & \\
\hline 5 & Almoloya de Alquisiras & 1757 \\
\hline 28 & Pachuquilla & 1017 \\
\hline 38 & Plan de Vigas & 905 \\
\hline \multirow[t]{2}{*}{30} & San Andrés Tepetitlán & 1001 \\
\hline & Amatepec & \\
\hline 6 & Amatepec & 1729 \\
\hline \multirow[t]{2}{*}{4} & Palmar Chico & 1916 \\
\hline & Coatepec de Harinas & \\
\hline 27 & Acuitlapilco & 1041 \\
\hline 9 & Chicoltepec & 1614 \\
\hline 3 & Coatepec de Harinas & 2362 \\
\hline 26 & Llano Grande & 1055 \\
\hline 44 & San José del Progreso & 856 \\
\hline 8 & Segunda del Monte & 1657 \\
\hline \multirow[t]{2}{*}{14} & Vueltas, Las & 1379 \\
\hline & Otzoloapan & \\
\hline \multirow[t]{2}{*}{36} & Otzoloapan & 917 \\
\hline & San Simón de Guerrero & \\
\hline 48 & San Diego Cuentla & 518 \\
\hline \multirow[t]{2}{*}{24} & San Simón de Guerrero & 1102 \\
\hline & Sultepec & \\
\hline 45 & Metlaltepec & 841 \\
\hline 37 & San Miguel Totomaloya & 906 \\
\hline 16 & San Pedro Hueyahualco & 1240 \\
\hline 17 & Santa Cruz & 1236 \\
\hline 7 & Sultepec & 1724 \\
\hline \multirow[t]{2}{*}{11} & Sultepequito & 1542 \\
\hline & Tejupilco & \\
\hline 2 & Bejucos & 2468 \\
\hline 46 & Luvianos & 669 \\
\hline 49 & Nanchititla & 463 \\
\hline 19 & Ocotepec & 1181 \\
\hline 1 & Tejupilco & 9298 \\
\hline \multirow[t]{2}{*}{31} & Zacatepec & 987 \\
\hline & Temascaltepec & \\
\hline 42 & Carboneras & 864 \\
\hline 29 & Comunidad, La & 1015 \\
\hline
\end{tabular}




\begin{tabular}{clr} 
CUADRO 2 (Continuación) & \\
\hline Clave & Municipio/Localidad & Población \\
\hline 47 & Rincón de Tequisquipan & 521 \\
23 & Rincón de San Andrés de los Gama & 1116 \\
15 & San Francisco Oxtotipan & 1357 \\
39 & San Lucas del Pulque & 896 \\
33 & San Mateo Almoloya & 943 \\
32 & San Pedro Tenayac & 959 \\
13 & Temascaltepec & 1385 \\
& Texcatitlán & \\
22 & San Agustín & 1137 \\
35 & Santa María & 930 \\
25 & Texcapilla & 1061 \\
& Tlatlaya & \\
18 & Coatepec & 1236 \\
40 & Naranjo, El & 892 \\
41 & Nuevo Copaltepec & 868 \\
20 & San Antonio del Rosario & 1179 \\
10 & San Pedro Limón & 1573 \\
12 & Santa Ana Zicatesoyan & 1395 \\
& Zacazonapan & \\
21 & Zacazonapan & 1138 \\
& Zacualpan & \\
34 & Hitzoltepec & 934 \\
43 & Zacualpan & 859 \\
\hline
\end{tabular}

Fuente: Datos aún no publicados de la Integración Territorial del Estado de México, INEGI-SPP, 1980.

CUADRO 3

Localización de las unidades de servicio de salud rural concentrado (USSRC) y del Hospital General del Instituto de Salud (SSA) del Estado de México en la región de estudio, 1988

\begin{tabular}{rlcc}
\hline Clave & Localidad & Población & Unidad de Servicio \\
\hline 1 & Tejupilco & 9298 & Hospital General \\
2 & Bejucos & 2468 & USSRC \\
4 & Palmar Chico & 1916 & USSRC \\
6 & Amatepec & 1729 & USSRC \\
13 & Temascaltepec & 1385 & USSRC \\
43 & Zacualpan & 859 & USSRC \\
46 & Luvianos & 669 & USSRC
\end{tabular}

Fuente: Localización de las unidades de servicios de salud del Instituto de Salud (SSA) del Estado de México, 1988 (mimeografiado). 


\section{CUADRO 4}

Comparación de las distancias recorridas por la demanda potencial para acceder a la unidad de servicios de salud más cercana, según su localización real y óptima en la región de estudio

\begin{tabular}{lcccc}
\hline Distancia & $\begin{array}{c}\text { Real } \\
\text { (a) }\end{array}$ & $\begin{array}{c}\text { Optima } \\
\text { (b) }\end{array}$ & $\begin{array}{c}\text { Diferencia } \\
\text { absoluta } \\
(a-c)\end{array}$ & $\begin{array}{c}\text { Grado de } \\
\text { ineficiencia } \\
\text { (a/b) }\end{array}$ \\
\hline Total $^{1}$ & 2465988 & 1287469 & 1178519 & 1.92 \\
Promedio por habitante $^{2}$ & 43.6 & 19.0 & 26.6 & 2.29 \\
\hline
\end{tabular}

Fuente: cálculos propios.

${ }^{1}$ Es la suma de los recorridos de toda la demanda potencial considerada (la población multiplicada por la distancia) a las unidades de servicios de salud más próximas, según el patrón real de localización de la oferta (c) y según el patrón de localización de la oferta definido por el modelo (b).

${ }^{2}$ Es la distancia promedio que recorrería cada habitante para acceder a la unidad de servicios de salud más próxima, según el patrón real de localización de la oferta (o) y según el patrón de localización de la oferta definido por el modelo (c).

te de $43.6 \mathrm{~km}$, frente a $19.0 \mathrm{~km}$ para el patrón óptimo; es decir, una diferencia absoluta de $24.6 \mathrm{~km}$ y relativa de $56.42 \%$ en cada viaje. Si además se considera la baja velocidad promedio de transporte en las áreas rurales y la, a menudo, urgente y vital necesidad de acceder a los servicios de salud, los datos anteriores adquieren mayor relevancia.

\section{Consideraciones finales}

Lo presentado anteriormente no pretende ser más que un intento por evaluar, de alguna manera, el patrón real de localización de uno de los múltiples servicios públicos con los que se intenta incidir en el bienestar y en el desarrollo de la población del estado de México, ${ }^{4}$ y sólo tiene el propósito de llamar la atención sobre la

4 N.B. Teitz (1968) ofrece una imagen muy clara de la importancia que tienen los servicios públicos para el bienestar de la población en las sociedades contemporáneas, cuando dice: “. . .el hombre moderno nace en un hospital financiado con recursos públicos, se transporta en un sistema de transporte público, se comunica a través de sistemas públicos o cuasipúblicos de comunicaciones, bebe su agua de una red de distribución pública, elimina sus desechos mediante el sistema de limpieza pública, lee los libros de sus bibliotecas públicas, disfruta y se recrea en sus parques públicos, es protegido por la fuerza pública y los cuerpos de seguridad pública, eventualmente morirá en un hospital público y aún podría ser enterrado en un cementerio público". 
necesidad de encontrar fórmulas y mecanismos de monitoreo $\mathrm{y}$ evaluación de las políticas públicas, como requisito para la práctica saludable de la planeación del desarrollo regional.

De los resultados obtenidos, son varios los aspectos que parecen requerir atención por parte de la oferta del servicio de salud pública:

a) La localización del Hospital General en Tejupilco es acertada. No sólo se ubica en el principal centro regional, sino que ofrece las mayores facilidades de acceso para la población.

b) Salvo la localización del Hospital General y de dos USSRC, el actual patrón de localización de las unidades de servicios de salud en la región no es el más adecuado en términos de su accesibilidad física. Esto exige una revisión cuidadosa de los criterios que guiarán las decisiones futuras de localización de los nuevos elementos que, eventualmente, se incorporarán al sistema regional de salud pública.

c) Se identifican dos áreas estratégicas en términos de la accesibilidad total del sistema, que requieren particular atención: una al este de la región de estudio y la otra en el extremo sur. La primera presenta una alta densidad de asentamientos, por lo que la oferta del servicio debería serle incrementada, y la segunda, aislada del resto de la región (y del estado), podría muy bien considerarse como punto de oferta en el futuro.

Los datos generados a partir de la aplicación del modelo de localización deben tomarse con cautela. El modelo utilizado, como todos los modelos, es una abstracción y una simplificación de la realidad que pretende representar. No considera todas las variables pertinentes para la solución del problema y simplemente es una herramienta auxiliar para la evaluación de una política pública de localización de servicios. Por lo tanto, los resultados deben tomarse en un sentido más cualitativo que cuantitativo y a la luz de las consideraciones y supuestos del modelo. Sólo así se puede entender la utilidad y bondades del manejo de los modelos matemáticos en la planificación regional.

Finalmente, habría que decir que en la medida en que modelos como el utilizado en este trabajo (aún simples herramientas de análisis regional) se perfeccionen en su diseño, construcción y utilización, e incorporen un mayor número de variables, en esa misma medida:

1. Serán más útiles en el diseño y evaluación de las políticas de localización de los servicios públicos.

2. Serán un instrumento que ayude efectivamente a tomar decisiones más racionales y menos intuitivas en la planificación del desarrollo regional. 
3. Favorecerán el uso exitoso de los recursos públicos de acuerdo con las mejores aspiraciones de la sociedad.

\section{Bibliografía}

Askew, I. (1983), "The location of service facilities in rural areas: a model for generating and evaluating, alternative solutions", Reg. Studies, vol. 17 , núm. 5 .

Boulding, K. (1966), "The concept of need for health services", Milbank Memorial Fund Quarterly, 44, 202-223.

Bradshaw, J. (1972), "The concept of social need", New Society, 30 March, 640-643.

Camposortega, S. y M.A. Mejia (1988), La marginación en el Estado de México: un aporte a la planificación del desarrollo, El Colegio Mexiquense.

De Jung, G. y F. Rutten (1983), "Justice and health for all", Social Science and Medicine, vol. 17, 16C, 1 085-1 095.

Doyal, L. (1979), The political Economy of health, Pluto Press.

Eyles, J. y K.J. Woods (1983), The social Geography of Medicine and Health, Londres: Croom Helm.

Fein, R. (1967), The doctor shortage: economic diagnosis, Brookings Institute, EUA.

Garrocho, C. (1989), Estructura funcional del sistema de asentamientos del Estado de México, Serie Cuadernos de Trabajo, El Colegio Mexiquense.

Graizbord, B. y C. Garrocho (1988), Sistemas de ciudades: fundamentos teóricos y operativos, El Colegio Mexiquense, Serie de Cuadernos de Trabajo, 1988.

Hagerstrand, T. (1970), "What about people in regional science?", PPRSA, 24.

Harvey, D. (1977), Urbanismo y desigualdad social, Siglo XXI, Madrid.

Hayes, M. (1986), "Your good health: access to health care in Northern Ireland, Beg. Studies, 20.

INEGI-SPP (1987), Estado de México: cuaderno de información para la planeación, INEGI-SPP, México.

Ingram, D. (1977), "The concept of accessibility: a search for an operational form", Reg. Studies, 5.

Instituto de Salud del Estado de México (ISEM) (1988), Dotación de servicios, equipamiento y personal médico por municipio (Documento en mecanografía).

Localización de las unidades de servicios de salud del Instituto de Salud (ISEM) del Estado de México (1988) (documento en mecanografía).

Joseph, A. y P. Bantock (1982), "Measuring potential physical accessibility to General Practitioners in rural areas: a method and a case study", Social Science and Medicine, 16D, 85-90. 
López Acuña, D. (1982), La salud desigual en México, Siglo XXI. Editores

Knox, P. (1979), "The accessibility of primary care to urban patients: a geographical analysis", lournal of the Royal College of General Practitioners, 29, 160-168.

Mburu, F.M. (1983), "Health systems as defences against the consequences of poverty: equity in health as social justice", Social Science and Medicine, vol. 17, $16 \mathrm{H}, 1149-1157$.

Mosley, M. (1979), Accessibility: the rural challenge, Methuen, London.

Musgrave, R. A. (1958), The theory of public finance, Mc Graw-Hill, N.Y.

Navarro, V. (1976), Medicine under capitalism, Prodist.

Oppenheim, N. (1980), Applied models in urban and regional annalysis, Englewood Cliffs, N.: Prentice Hall, Inc.

Phillips, D.R. (1979), "Spatial variations in attendance at general practitioners services", Social Science and Medicine, 13D, 169-181.

(1981), Contemporary issues in the Geography of health care, Norwich: Geo Books.

Pinch, S. (1985), Cities and services, Rutledge and Kegan Paul Ltd., Londres.

Rodriguez, F. (1988), Distribución del nivel de vida municipal y satisfacción de las necesidades básicas en el Estado de México: 1960-1980, Serie Cuadernos de Trabajo, El Colegio Mexiquense, 1988.

Rushton, G. (1979), Optimal location of facilities, Wentworth, N.H., Compress.

Samuelson, P.A. (1954), "The theory of public expenditures", The Review of Economics and Statistics, vol. 36.

(1955), "Diagramatic exposition of a theory of public expenditure", The Review of Economics and Statistics, vol. 37.

Smith, D.M. (1979), Where the grass is greener: living in an unequal world, Harmonsdsworth, Penguin.

Stimnson, R. (1981), "The provision and use of General Practitioner services in Adelaide, Australia: application of tools of location analysis and theories of provider and user spatial behaviour", Social Science and Medicine, 15D, 27-44.

Teitz, N.B. (1968), "Toward a theory of urban public facility location", $\mathrm{Pa}$ pers of the Regional Science Association, 21, 35-51.

Townsand, P. (1981), "Toward equality in health through social policy", International Journal of Health Serviçes, 11, 63-75.

(1982), y N. Davidson, Inequalities in health: the Black Report, Penguin Books, Harmondsworth.

Turner, B.S. (1986), Inequality, Ellis Horwood Ltd, Inglaterra.

Veatch, R.M. (1981), Ethical aspects of the right to health care, en Hiller M.D. (comp.), Medical ethics and the law, Cambridge, MA.

Ward, P. (1986), Welfare politics in México: papering over the cracks, Allen and Unwin, Ltd, Londres. 